\title{
Mapping the global mRNA transcriptome during development of the murine first molar
}

\section{Maria A. Landin ${ }^{1 *}$, Ståle Nygård ${ }^{2}$, Maziar G. Shabestari ${ }^{3}$, Eshrat Babaie ${ }^{4}$, Janne E. Reseland ${ }^{3}$ and Harald Osmundsen ${ }^{1}$}

${ }^{1}$ Department of Oral Biology, Faculty of Dentistry, University of Oslo, Oslo, Norway

${ }^{2}$ Bioinformatics Core Facility, Institute for Medical Informatics, Oslo University Hospital and University of Oslo, Oslo, Norway

${ }^{3}$ Department of Biomaterials, Institute for Clinical Dentistry, University of Oslo, Oslo, Norway

${ }^{4}$ The Biotechnology Centre of Oslo, University of Oslo, Oslo, Norway

\section{Edited by:}

Natalia Polouliakh, Sony Computer

Science Laboratories Inc., Japan

\section{Reviewed by:}

Ellis Fok, McGill University, Canada

Georges Nemer, American

University of Beirut, Lebanon

\section{*Correspondence:}

Maria A. Landin, Department of Oral Biology, Faculty of Dentistry, University of Oslo, Gjetmyrsvei 69-71, 0372 Oslo, Norway e-mail:mariaal@odont.uio.no; dosantla@online.no
The main objective of this study was to map global gene expression in order to provide information about the populations of mRNA species participating in murine tooth development at $24 \mathrm{~h}$ intervals, starting at the 11th embryonic day (E11.5) up to the 7th post-natal day (P7). The levels of RNA species expressed during murine tooth development were mesured using a total of 58 deoxyoligonucleotide microarrays. Microarray data was validated using real-time RT-PCR. Differentially expressed genes ( $p<$ 0.05 ) were subjected to bioinformatic analysis to identify cellular activities significantly associated with these genes. Using ANOVA the microarray data yielded 4362 genes as being differentially expressed from the 11th embryonic day (E11.5) up to 7 days post-natal (P7), 1921 of these being genes without known functions. The remaining 2441 genes were subjected to further statistical analysis using a supervised procedure. Bioinformatic analysis results for each time-point studied suggests that the main molecular functions associated with genes expressed at the early pre-natal stages (E12.5E18.5) were cell cycle progression, cell morphology, lipid metabolism, cellular growth, proliferation, senescence and apoptosis, whereas most genes expressed at post-natal and secretory stages (P0-P7) were significantly associated with regulation of cell migration, biosynthesis, differentiation, oxidative stress, polarization and cell death. Differentially expressed genes (DE) not described earlier during murine tooth development; Inositol 1, 4, 5-triphosphate receptor 3 (Itpr3), metallothionein 1(Mt1), cyclin-dependent kinase 4 (Cdk4), cathepsin D (Ctsd), keratin complex 2, basic, gene 6a (Krt2-6a), cofilin 1, non-muscle (Cf/1), cyclin 2 (Ccnd2), were verified by real-time RT-PCR.

Keywords: gene expression, microarrays, metallothionein 1, ITPR3, qPCR, tooth development

\section{INTRODUCTION}

During murine tooth development substantial changes occur within a time-span of $24 \mathrm{~h}$. From E1 1.5 up to P7, i.e., in the course of 16 days the tooth germ develops from one layered oral epithelium into a phenotypic molar tooth. The developing murine tooth germ is therefore an excellent model for studying the time-course of gene expression in a rapidly, developing organ.

Differentiation requires participation of a large number of genes, the expression of which is regulated in time and space. The numbers and types of genes involved, however, vary depending on the developmental stage. In addition, miRNAs and lncRNAs are also known to be involved during tooth development (Jevnaker and Osmundsen, 2008; Michon et al., 2010; Michon, 2011) providing an additional layer of complexity.

Microarray studies generate vast amounts of gene expression data. Genes that exhibit significantly different levels of expression at different developmental stages during tooth development are termed differentially expressed (DE) genes; these must be isolated using appropriate statistical methods (Reiner et al., 2003; Smyth,
2004). Secondly it is of interest to determine biological processes, biochemical functions, and sub-cellular locations significantly associated with DE genes, linking DE genes to alterations in cellular physiology by mapping DE genes to gene ontology (GO) terms (Ashburner et al., 2000; Rhee et al., 2008).

More than 4300 mRNAs and some of their translated proteins have, so far, been detected during tooth germ development by microarray, in situ hybridization and immunocytochemistry (Jevnaker and Osmundsen, 2008; Landin et al., 2012). In a previous study (Landin et al., 2012) we used three pre-selected genes (Ambn, Amelx, and Enam) as starting point for profile search and identified 84 differentially expressed genes with a similar expression pattern as these enamel genes. However, the results of this study only show a tinny frame of the big picture of genetic events that occur during murine tooth development. Mapping global gene expression to capture the majority of genes involved during murine tooth development may provide an overview of the occurring genetic changes.

The global mapping of gene expression for each timepoint studied may also reveal participation of novel genes or 
transcription factors as well as genes with unknown functions (Etokebe et al., 2009) during murine tooth development. Understanding the molecular cell biology during murine tooth development opens for development in novel bio-therapeutic strategies in dentistry.

In the current study we attempted to map the global gene expression in the molar murine tooth germ at each of 16 timepoints by uploading the 2441 genes differentially $(P \leq 0.05)$ expressed at every time point studied (E11.5-P7). To interpret the resulting gene-expression data, we used Ingenuity Pathway analysis (IPA) (Kramer et al., 2014).

\section{METHODS}

\section{EXPERIMENTAL DESIGN}

The global gene expression in tooth germs from wild type mice was monitored from embryonic day 11 (E11.5) up to 7 days after birth (P7) using a reference design; the 11th embryonic day was considered time point zero $\left(\mathrm{T}_{0}\right)$ and all time-points studied were compared to E11.5. The sample size for each time point was $n=3-5$ embryos/pups from three different mothers.

\section{EXPERIMENTAL ANIMALS}

Pregnant Balb-c mice CD-1strain were used in this study. The day of vaginal plug was set to E0.5 Adult mice were sacrificed by cervical dislocation, the embryos or pups by decapitation. Embryos were staged according to the Theiler criteria as described in Landin et al. (2012). Animal housing (Scantainer ventilated cabinet Q-110) had $12 \mathrm{~h}$ light/dark cycle. The cabinet temperature was maintained at $21^{\circ} \mathrm{C}$ with a relative humidity of $55 \%$ (ScanClime plus). Fodder and water were supplied ad lib. The animals were kept according to the regulations of the Norwegian Gene Technology Act of 1994.

\section{DISSECTION OF TOOTH GERMS AND RNA EXTRACTION}

Dissection, homogenization of whole tooth germs and total RNA extraction was carried out as previously described in Osmundsen et al. (2007) and Landin et al. (2012). At the pre-natal stages total RNA was isolated from 3 to 5 tooth germs. At post-natal stages batches of at least three tooth germs were used at each time point. RNA concentration was measured at $260 \mathrm{~nm}$ in a Nanodrop ND 1000 spectrophotometer. RNA fractions with the ratio of absorbance 260 and $280 \mathrm{~nm}$ around 2.0 and with RINvalues higher than 8.5 as measured using an Agilent Bioanalyzer (Agilent, Palo Alto, CA, USA) were used for analysis of gene expression using deoxyoligonucleotide microarrays and real-time RT-PCR.

\section{COMPLEMENTARY DNA SYNTHESIS AND LABELING}

Complementary DNA (cDNA) was synthetized and indirect labeled from $1 \mu \mathrm{g}$ total RNA using Genisphere Array $900^{\mathrm{TM}}$. Indirect labeling was used to avoid bias associated with differences in molecular size of the indicator molecules as previously described (Osmundsen et al., 2007; Landin et al., 2012).

\section{MICROARRAY ANALYSIS OF mRNAs ISOLATED FROM TOOTH GERMS}

Murine deoxyoligonucleotide (30 k)-microarrays were purchased from the NTNU Microarray Core Facility, Trondheim, Norway. The slides had been printed using the Operon murine v. 3 oligo set
(Qiagen GmbH, Hilden Germany). The arrays included probes for 10 different mRNAs from Arabidopsis thaliana. A spike mixture of 10 different mRNAs from A. thaliana (purchased from Stratagene, La Jolla, CA, USA) mixed in pairs at 10 different ratios, ranging from 0.1 to 5 was used to monitor the quality of the hybridisation. Each time point 3-6 biological replicates were subjected to independent microarray analysis. Each microarray was scanned in a Packard Bioscience Scanarray Lite microarray scanner (Perkin-Elmer). The Cy3 (543 nm) and Cy5 (645 nm) fluorescence signals were quantified by using the ScanArrayExpress v.3.0 software (Perkin-Elmer).

\section{ACCESSION NUMBER}

Raw and normalized microarray data have been deposited in the ArrayExpress database with reference E-MEX-3581.

\section{MICROARRAY DATA PREPARATION AND NORMALIZATION}

For each microarray, measured net fluorescence intensities (median values, with background subtracted) were Lowess normalized. Spots with net intensity less than 200 across the entire time-course were filtered away. The filtered data were $\log _{2}$ transformed and subjected to median subtraction and z-score normalization (Quackenbush, 2002; Cheadle et al., 2003).

\section{STATISTICAL ANALYSIS OF MICROARRAY DATA}

To find non-constant (non-zero) genes expressed during murine tooth development (E11.5-P7), statistical analysis of microarray data was carried out, using Spotfire v. 9 Microarray Analysis Software (TIBCO Software Inc, Palo alto, CAL, USA). Microarray data was derived from sets of three to six arrays at each time point. Data from a total of 58 arrays were combined into a single data file and treated as single color data to facilitate statistical analysis of time-courses. False discovery rate (FDR; 0.05) of Benjamini and Hochberg (Benjamini et al., 2001), was used to correct selection of genes for false positives. The ANOVA facility of the Spitfire program was used to select genes which exhibited statistically significant differences in levels of expression $(P<0.05)$ between the various developmental stages.

\section{VALIDATION OF MICROARRAY RESULTS USING REAL-TIME RT-PCR}

Expression of selected DE genes: Inositol 1, 4, 5-triphosphate receptor 3 (Itpr3), metallothionein 1(Mt1), cyclin-dependent kinase 4 (Cdk4), cathepsin D (Ctsd), keratin complex 2, basic, gene $6 a$ (Krt2-6a), cofilin 1, non-muscle (Cfl1), cyclin 2 (Ccnd2), were verified by real-time quantitative RT-PCR. These genes were selected because they are not described in literatur during tooth murine tooth development. The assays were carried out as described previously (Osmundsen et al., 2007; Landin et al., 2012) using Stratagene MX 3005P PCR instrument (Stratagene, La Jolla, CA, USA) using both biological and technical triplicates. The spesific primers are listed in Table 1. Statistical evaluation of the significance of differences between measured $C t$-values was carried out using the REST 2009 program (Pfaffl et al., 2002a,b).

\section{BIOINFORMATICS ANALYSIS OF DIFFERENTIALLY EXPRESSED GENES}

The 2441 differentially expressed genes with known Entrez Gene ID at all time- points, were uploaded onto IPA (Ingenuity Systems 
Table 1 | Sequences of deoxyoligonucleotide primers used for real-time RT-PCR assays.

\begin{tabular}{|c|c|c|}
\hline Gene & Sequence of left primer & Sequence of right primer \\
\hline Itp3 & 5'-ATG CTG CAG GCC TAT GAG GAG-3' & 5'-TAC AGA CTG CTT GCG GCT CAG-3' \\
\hline Mt1 & $5^{\prime}$-CAG GGC TGT GTC TGC AAA G-3' & 5'-GCT GGG TTG GTC CGA TAC TA-3' \\
\hline Ctsd & 5'-CCA CTG TCA GGG AAC TGG AT-3' & 5'CTC CTT CAG ACA GGC AGA GG-3' \\
\hline Krt2-6a & 5'-AGG CTG CTG AAG GAG TAC CA-3' & 5'-TCA ACC TGC АCT ССТ СТC СТ-3' \\
\hline Lmna1 & 5'-AGG ACC TCG AGG CTC TTC TC-3' & 5'-CTC CTT CAG CGT CTG TAG CC-3' \\
\hline$R p L 27$ & 5'-GGG AAA GTG GTG GTG GTG CT-3' & $5^{\prime}$-CAC CAG GGC ATG GCT GTA AG-3' \\
\hline
\end{tabular}

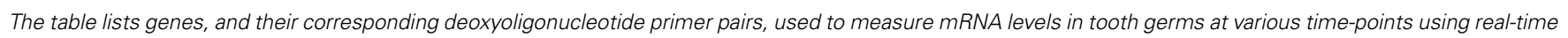
RT-PCR assays. Primers were designed to have melting point $60^{\circ} \mathrm{C}$ and product size of about $100 \mathrm{bp}$.

Inc., Redwood City, CAL, USA) (29). Bioinformatics core analysis was used to identify significant associations $(P \leq 0.05)$ with canonical pathways, signaling pathways and with molecular and cellular functions with clusters of differentially expressed genes as judged by Fisher's exact test. The core analysis was performed by uploading the ratios $\left(\mathrm{Time}_{\mathrm{n}} / \mathrm{E} 11.5\right)(n=\mathrm{E} 12.5-\mathrm{P} 7)$ of the global expression data directly from Spotfire. The identifier was the ENTREZ ID. The parameters chosen were: The reference set was the Ingenuity Knowledge Base (genes only), species [mouse, human and primary mouse cell culture (epithelial cells, odontoblasts, ameloblasts, and adipocytes)].

IPA Transcription Factor Analysis (Kramer et al., 2014) was used to identify the transcription factors associated with significant changes in gene expression during murine tooth development.

Network analysis (Thomas and Bonchev, 2010) was used to create graphical representations of molecules interacting at each time-point. The network size was set to 35 nodes/molecules. Network analysis also predicted the upstream and downstream effects of activation or inhibition on other molecules by applying expression values from the dataset (Supplementary data).

\section{RESULTS}

\section{GENES WITH EXPRESSION LEVEL CHANGING OVER TIME}

Microarray results showed that a total 4362 of non-constant (non-zero) genes are expressed during murine tooth development from E11.5 up to P7; 1921 genes with unknown function and very highly expressed at all time-points with net fluorescence intensities above 10000 (results not shown) and 2441 differentially expressed genes at all time- points with known Entrez Gene ID. These 2441 genes were subjected to further bioinformatics analysis.

\section{TIME COURSES OF EXPRESSION FOR SELECTED GENES AS ASSAYED USING REAL-TIME RT-PCR}

Time-course of expression of selected genes (Figure 1) was also monitored using real-time RT-PCR. The results suggest that time-courses assayed by real-time RT-PCR show a similar trend to expression data obtained using microarrays (Figure 2).

\section{BIOINFORMATICS ANALYSIS OF THE TIME-COURSE INGENUITY PATHWAY ANALYSIS (IPA) \\ Transcription factor analysis}

Transcription factor analysis suggested that 19 transcription factors are involved in the transcription of 23 genes during the invagination of the epithelium into the mesenchyme at E12.5 (Table 2). The transcription factor (TF) Huntingtin ( $\mathrm{Htt}$ ) in the tooth germ regulated other transcription factors e.g., Hifla, Purb, and C/ebp (Figure 3A).

At the early bud stage (E13.5) the number of transcription factors involved decreased to 6 compared to E12.5 (Figure 3B). In this early budding stage, transcription factor analysis show, that $\mathrm{V}$-mycavian mielocytomatosis viral oncogene homolog $(m y c)$ regulated other transcription factors (Hif1A, Eno1, Eif4g1, Eif4A1, $I d 1, I d 2$, and Hmgal) (Figure 3B). Myc seems also to be regulated by Drap1, Hox9, and Hmgal. In addition Hmgal regulated the transcription factors $I d 3$ and Trim 28 (Figure 3B).

During the formation of the enamel knot (E14.5) the number of transcription factors decreased to 3 compared to E12.5-E13.5 (Figure 3C). At this embryonic stage of tooth development, myc seems to regulate the transcription factors Hifla, Smarcc1, and Mycn. Mycn regulates Mxl1 (Figure 3C).

At the early bell stage (E15.5) both the number transcription factors and genes regulated by TF increased (Figure 3D). Transcription factor analysis suggests that Tp53, Ahr and Hoxa10, may play an important role at this stage of tooth development. Tp53 is regulated by Ahr and Hoxal0 and in turn regulates Bag1, Ncor2, Actg2, and Hmgal (Figure 3D). During bell stages (E16.5-E17.5) the number of transcription factors decreased (Figures 3E,F) compared to E15.5 (Figures 3D,E). Hmgal regulated Id3, Trim28, and Sox4, while Drap1 regulates Ilf2 (Figure 3E).

At the late bell stage (E18.5) (Figure 3G) and post-natal stages (P0-P7) (Figures 3H-O) the number of transcription factors remains almost constant.

\section{Network analysis}

Genes expressed during placode formation (E12.5) were associated with network functions such as post-translational modification, cellular growth and proliferation, cell cycle and cell-to-cell signaling (Table 4 and Supplemental data pages 3-6). 

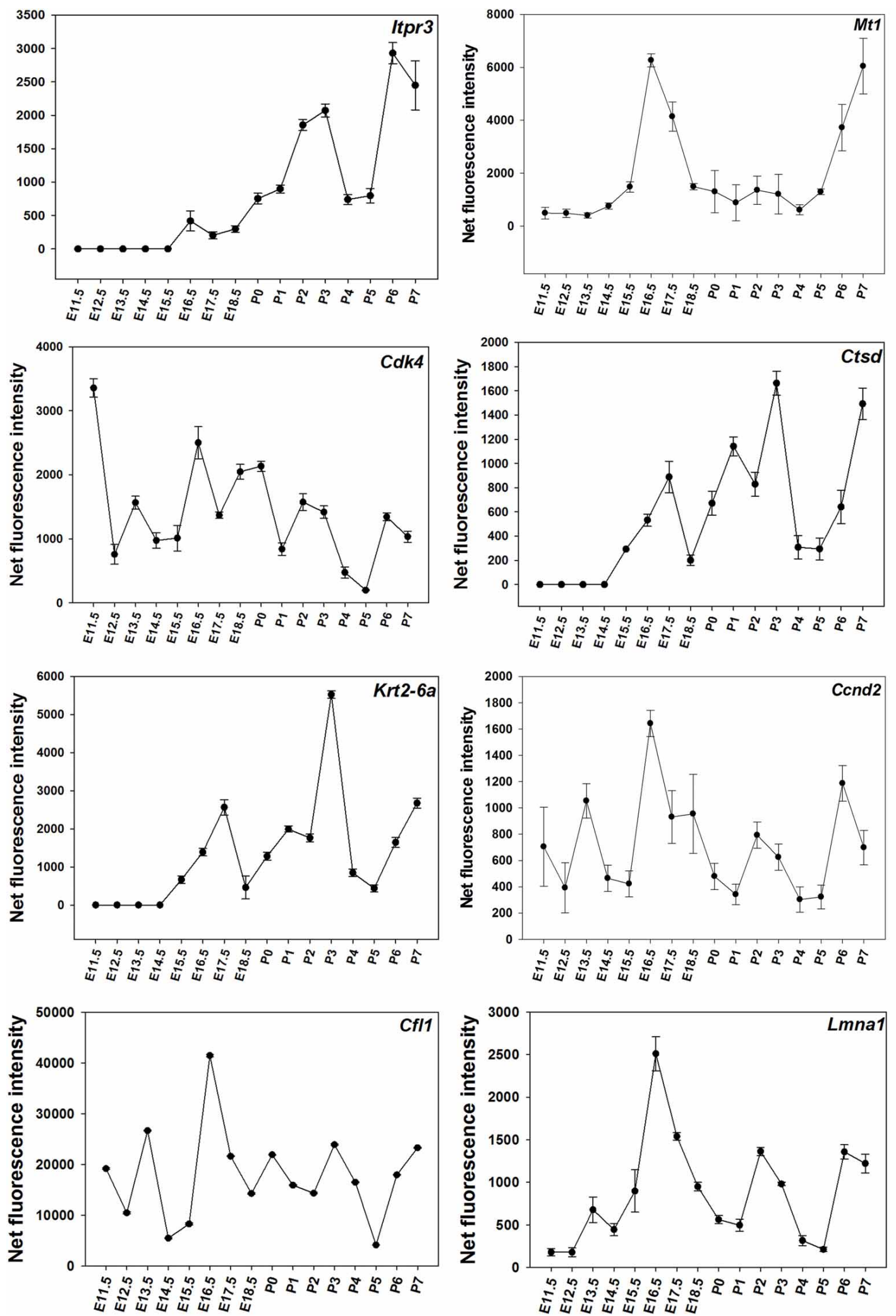

FIGURE 1 | Microarray results for for DE genes throughout the time-course. The figure presents results showing the net normalized fluorescence intensities for DE genes monitored using microarrays with SD. 

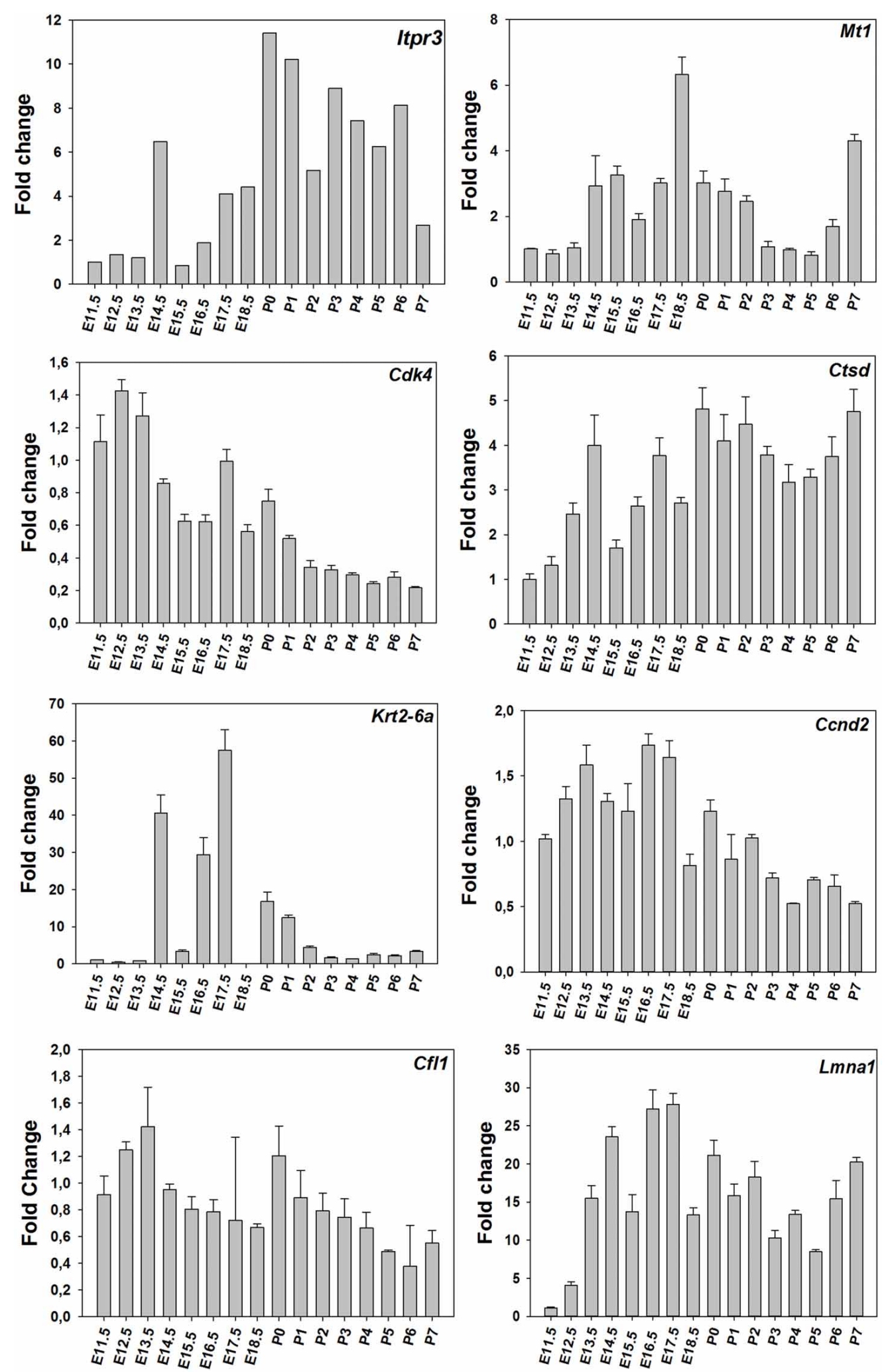

FIGURE 2 | RT-PCR results for the time-course. Levels of selected mRNAs in total RNA isolated from the molar tooth germ at the various times of development using real-time RT-PCR. 
Table 2 | Bioinformatic results for transcription factor analysis using IPA.

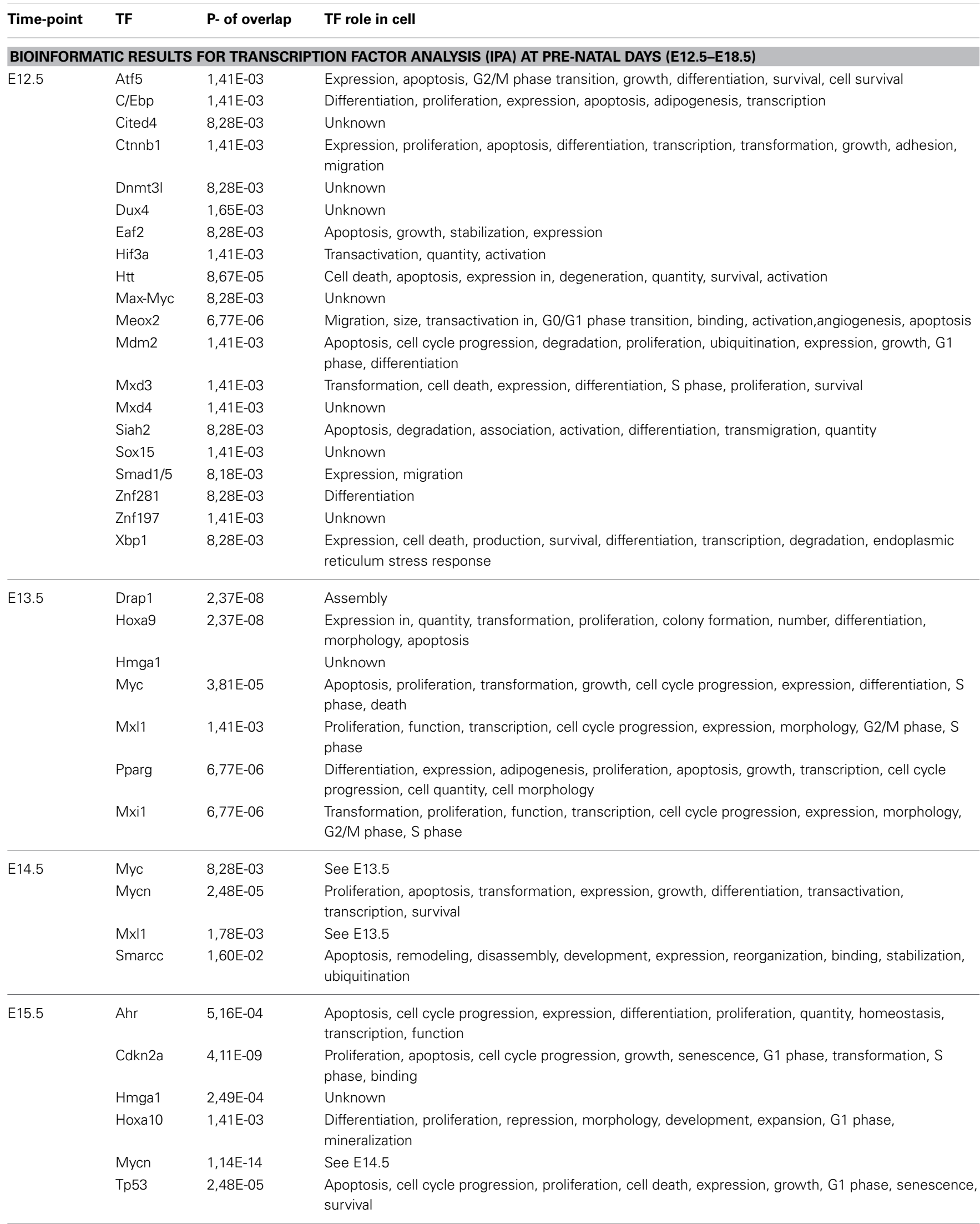


Table 2 | Continued

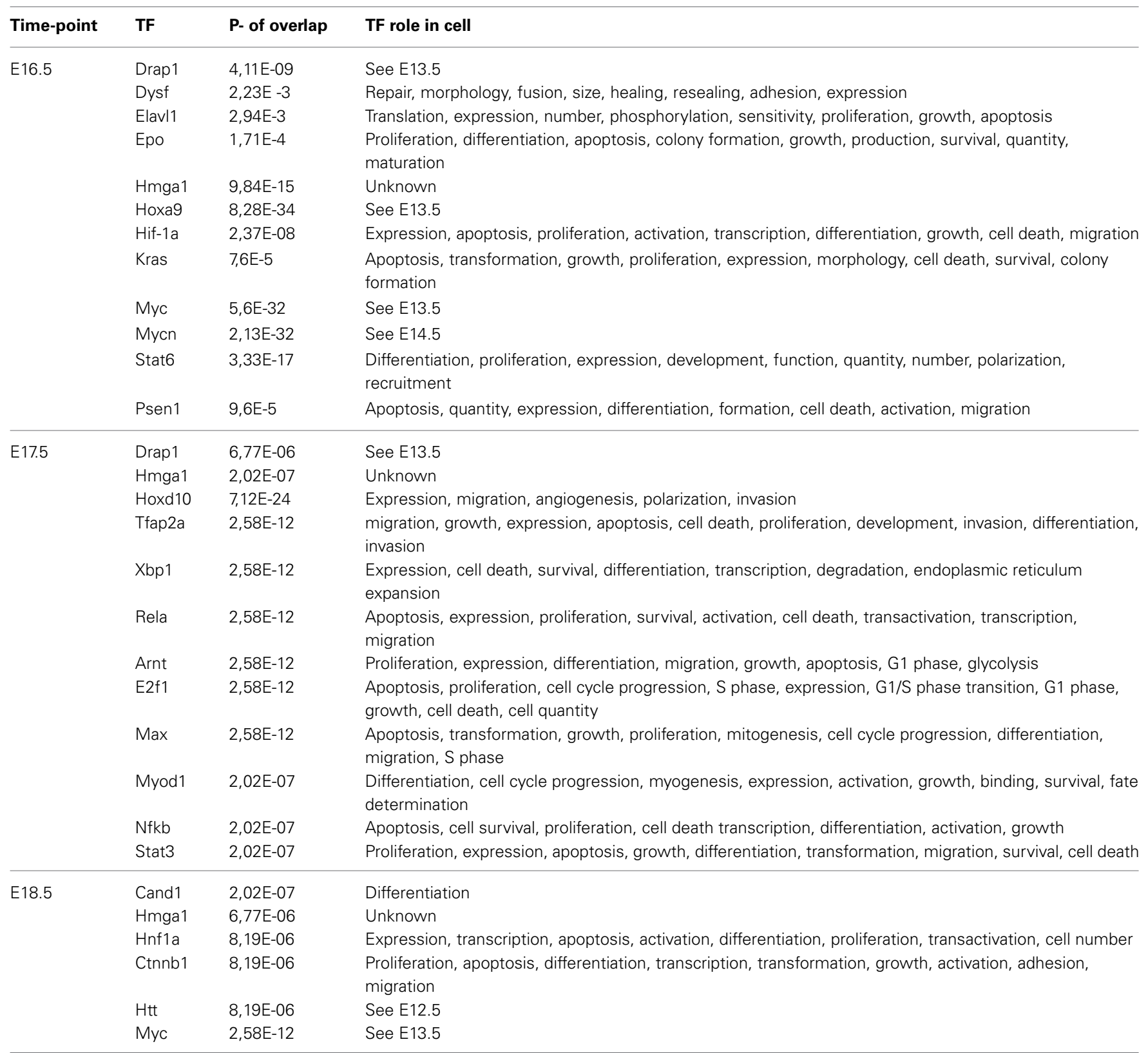

\begin{tabular}{|c|c|c|c|}
\hline \multicolumn{4}{|c|}{ BIOINFORMATIC RESULTS FOR TRANSCRIPTION FACTOR ANALYSIS (IPA) AT POST NATAL DAYS (P0-P7) } \\
\hline \multirow[t]{6}{*}{ PO } & Myc & $2,83 E-24$ & See E13.5 \\
\hline & Stat6 & $8,05 \mathrm{E}-12$ & See E16.5 \\
\hline & Tfap2a & 1,64E-07 & $\begin{array}{l}\text { Migration, growth, expression, apoptosis, cell death, proliferation, development, invasion, differentiation, } \\
\text { invasion }\end{array}$ \\
\hline & Hoxd10 & $4,00 \mathrm{E}-07$ & Expression, migration, angiogenesis, polarization, invasion \\
\hline & Mxl1 & 2,67E-05 & See E13.5 \\
\hline & Foxo1 & 2,64E-07 & $\begin{array}{l}\text { Expression, transcription, apoptosis, transactivation, proliferation, activation, differentiation, binding, } \\
\text { downregulation, ubiquitination }\end{array}$ \\
\hline \multirow[t]{4}{*}{ P1 } & Ahr & $6,05 \mathrm{E}-10$ & $\begin{array}{l}\text { Apoptosis, cell cycle progression, expression, differentiation, proliferation, quantity, homeostasis, } \\
\text { morphology, transcription, function }\end{array}$ \\
\hline & Esrra & $1,00 \mathrm{E}-07$ & Differentiation, expression, number, proliferation, growth, migration, ossification, uptake, glycolysis \\
\hline & Hmga1 & $6,16 \mathrm{E}-15$ & Unknown \\
\hline & Mycn & $2,60 \mathrm{E}-07$ & See E16.5 \\
\hline
\end{tabular}


Table 2 | Continued

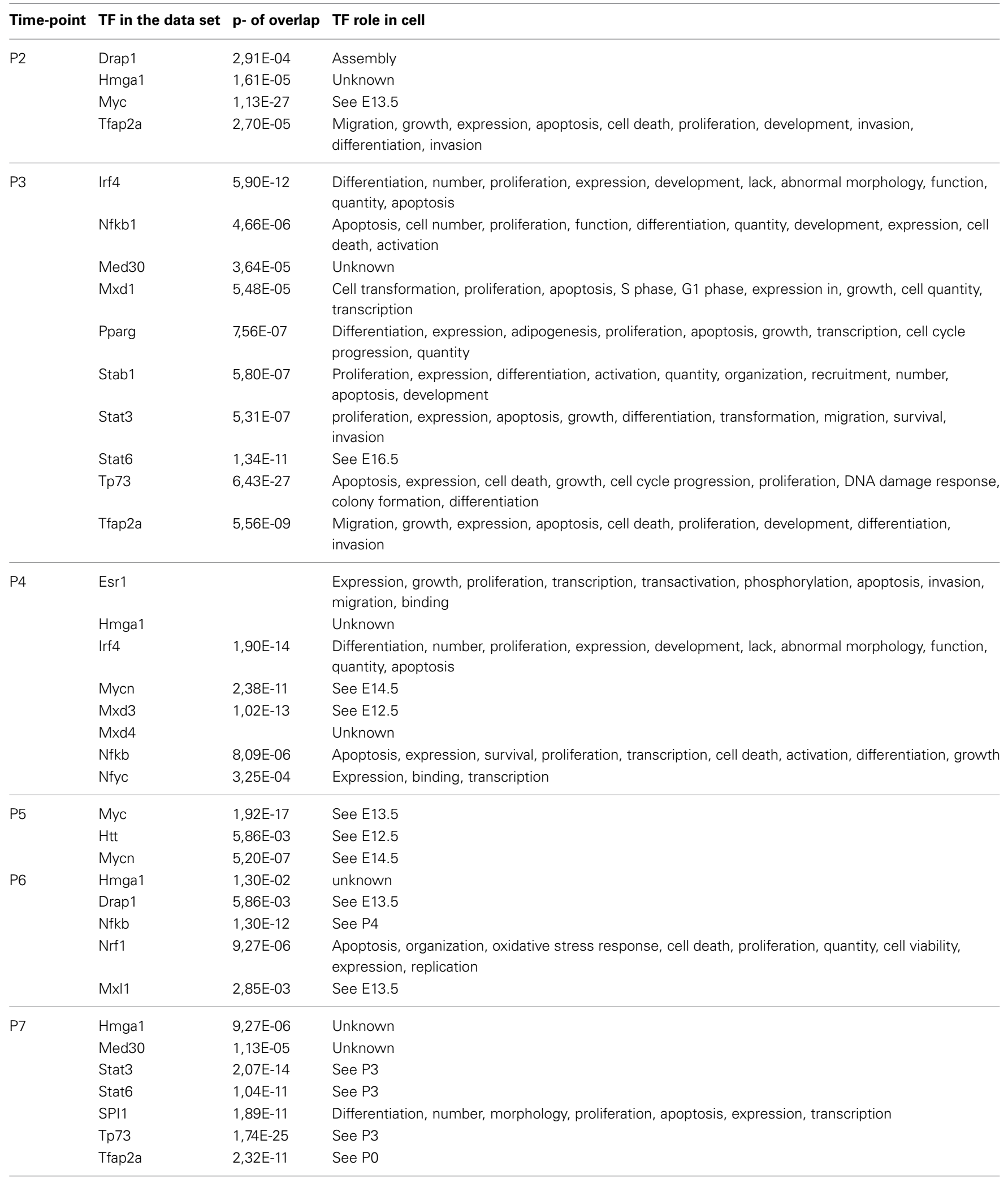

The 2441 differentially expressed genes (DE) ( $p \leq 0.05)$ were used to determine significant associations ( $p \leq 0.01)$ with transcription factors (TF) prior to birth. TFs with p-value of overlap $<0.01$ where considered to be significantly associated with the DE genes from the dataset.

Abreviations: TF, Transcription factors; embryonic day (E12.5-E18.5). 
A

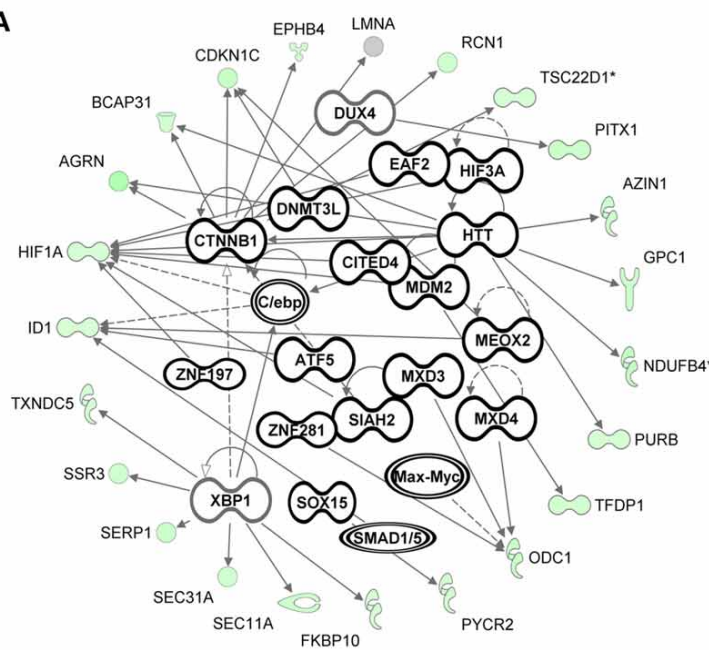

B

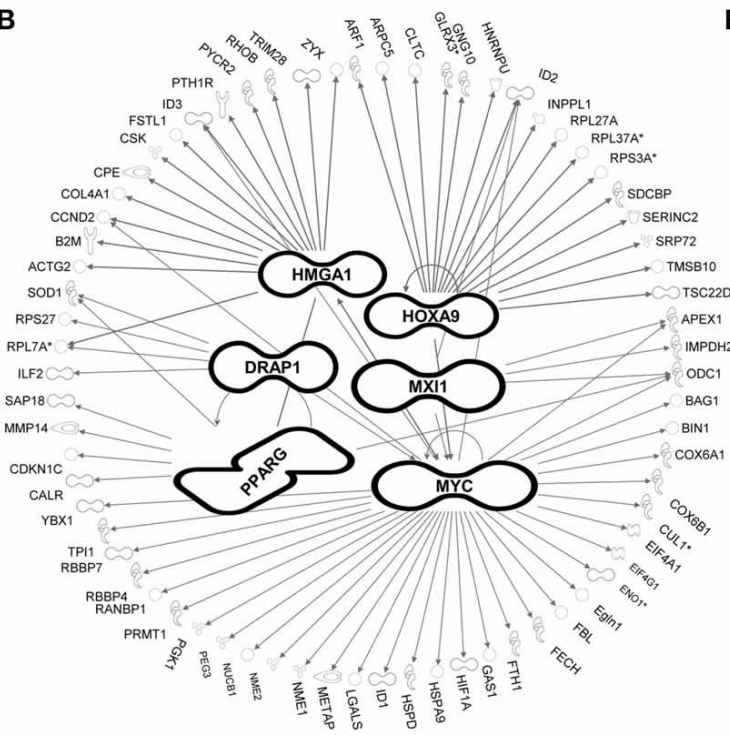

C

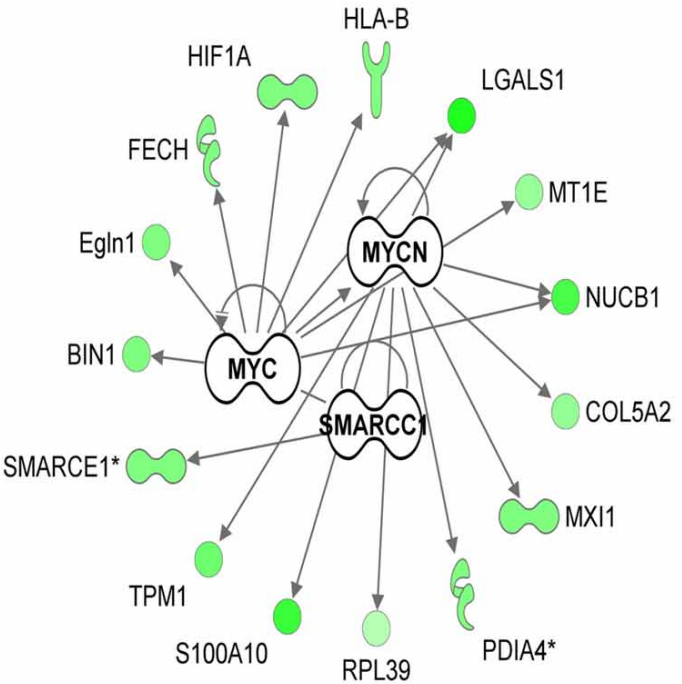

D

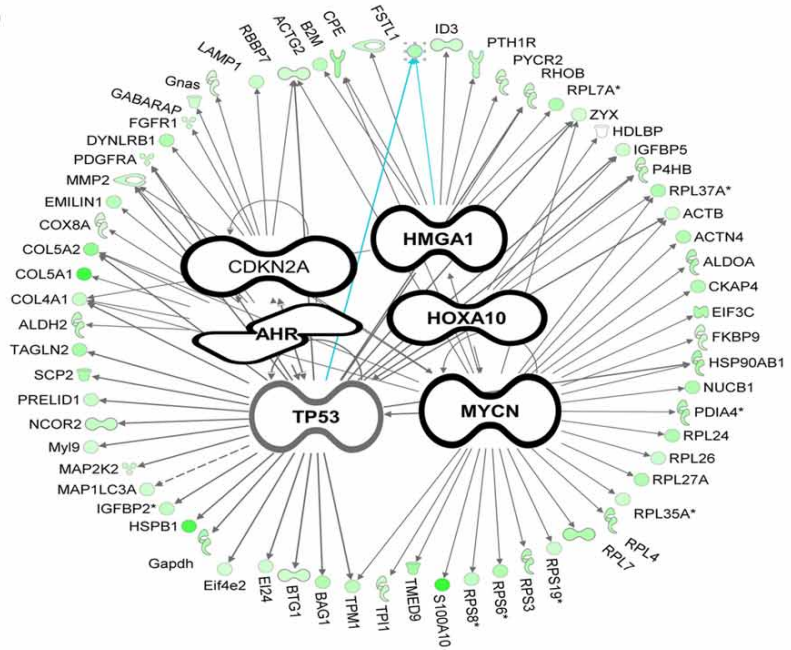

E

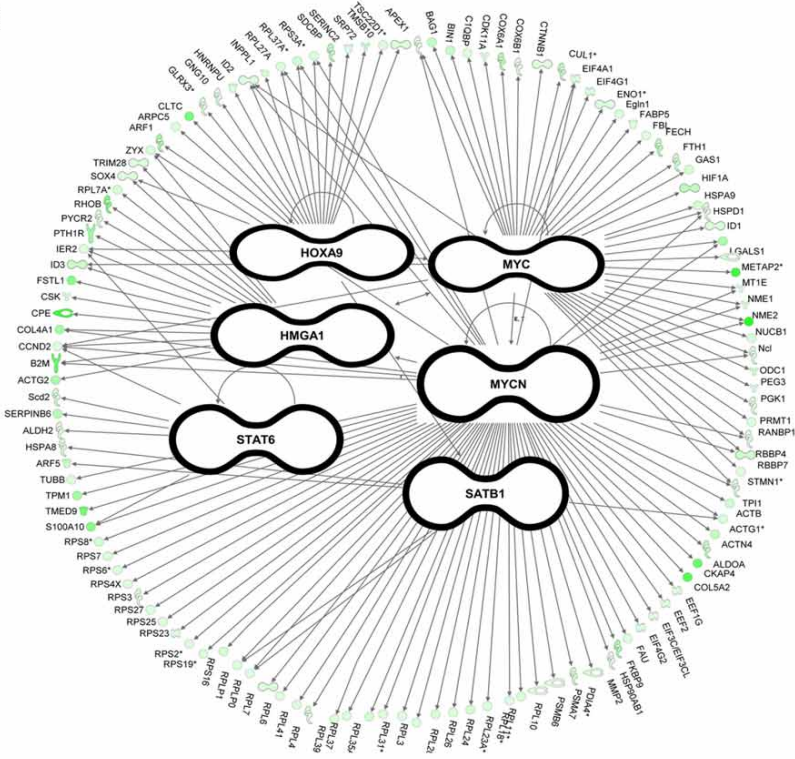

$\mathbf{F}$

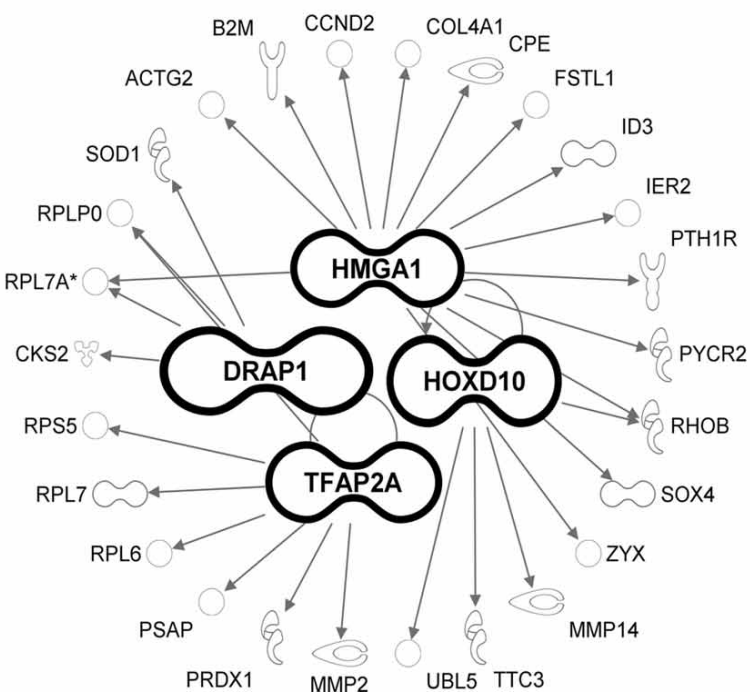

FIGURE 3 | Continued 
During bud formation (E13.5) the genes expressed at this stage were associated with the following network functions: Posttranscriptional modification, protein synthesis and folding, cellular compromise, cell death (Table 4 and Supplemental data pages 7-23).

The genes expressed at enamel knot (E14.5) Table 4 and Supplemental data pages 24-32) and late cap stages (E15.5E16.5), were associated with networks associated with functions listed in Table 4 and Supplemental data pages 33-52).

Genes expressed at bell stages E17.5-E18.5 were significantly associated with networks connected to lipid metabolism, nucleic acid and carbohydrate metabolism, small molecule biochemistry, senescence and energy production (Table 4 and Supplemental data pages 53-82).

Genes expressed at the post-natal stages (P0-P7) were associated with networks involved in DNA/RNA replication, cell-to-cell signaling, protein synthesis, cell death and free radical scavenging (Table 4 and Supplemental data pages 84-197).

\section{Biological functions and Canonical pathways}

Bioinformatics analysis of the global gene expression significantly $(p=0.05)$ associated the 2441 differentially genes $(p \leq$ $0.05)$ of the dataset with the molecular and cellular functions: "Gene expression" (all time-points studied) (Figures 4A-F), "cellular growth and proliferation" (E12.5-E13.5) (Figure 4A), "RNA post-transcriptional modification" (E-12.5-E13.5 and P0-P7) (Figures 4A,D-F), "cell cycle," "cell morphology" (E12.5-E14.5) (Figures 4A,B), "protein folding” (E12.5-E18.5) (Figures 4A-C), "nucleic acid metabolism" (E15.5-P7) (Figures 4B-F).

The canonical pathways associated with the $2441 \mathrm{DE}$ genes are listed in Table 3.

\section{DISCUSSION}

Transcription factor and network analysis of the 2441 differentially expressed genes suggests that during the embryonic stages of murine tooth development (E12.5-E13.5) cell proliferation and cell death are highly regulated e.g., expression of transcription regulators like Huntingtin (Htt), Hifla, Purb, Cbp1, C/ebp, $M y c$, and Id1. The epithelial and mesenchymal cells during bud- and bell-stages, proliferate, migrate, adhere and communicate through tight junction signaling as shown by the expression and regulation of $C c n d 2$ on the data set. $C c n d 2$ also plays a role in Wnt/ $\beta$-catenin signaling pathway (Liu and Millar, 2010). Wnt/ $\beta$ catenin is known to play a central role for the morphogenesis of ectodermal appendages such as teeth, hair and exocrine glands (Haara et al., 2012).

During early murine tooth development, ectodermal placodes proliferate and invaginate into the mesenchyme to form an epithelial bud (E12.5-E13.5). The proliferating epithelial cells form a controlled invasion front into the mesenchyme and hold a correct temporal and spatial pattern in the growing bud; this process requires genes that control migration and adhesion between cells e.g., Ctnnb1. Migration of epithelial cells into the underlying mesenchyme requires changes in the cytoskeleton enabling the epithelial cells to migrate and invade the mesenchyme. The cytoskeleton provides both structural scaffolding for cells and a transportation network, where particles move along microtubule and actin highways powered by molecular motors that burn the cellular fuel, ATP (Ikuta et al., 2014).

The number of cells in the growing epithelium bud/bell is also controlled either by apoptosis (Hif3a, Hifla, C/Ebp, Ctnnb1, Hoxa9, Hoxa10, Myc, and Eaf2) or arrest of cell cycle (Meox2, $M d m 2$ and Mxd3 and Mxi1) (E12.5-E18.5). Cyclin d2 (encoded by $C c n d 2$ ) is expressed in dental pulp and periodontal ligament cells (Liu et al., 2009). It is also known that Ccnd2 activates $C d k 4 / 6$, allowing the cells to progress through the G1-S checkpoint (Morsczeck et al., 2009). Epithelial cells also invade and displace the underlying mesenchyme. Mesenchymal cells probably use senescence as a trigger of tissue re-modeling in order to allow the epithelial cells to invade and replace mesenchymal tissue. Senescent cells arrest their own proliferation, recruit phagocytic immune cells and promote tissue renewal. Developmental senescence is well studied process during mammalian embryonic development (Munoz-Espin and Serrano, 2014). We can speculate that during early murine tooth development the epithelial cells at bud (E12.5-E13.5), cap (E14.5E15.5) and bell stages (E16.5-E18.5) migrate into the mesenchyme where senescence, followed by clearance and then

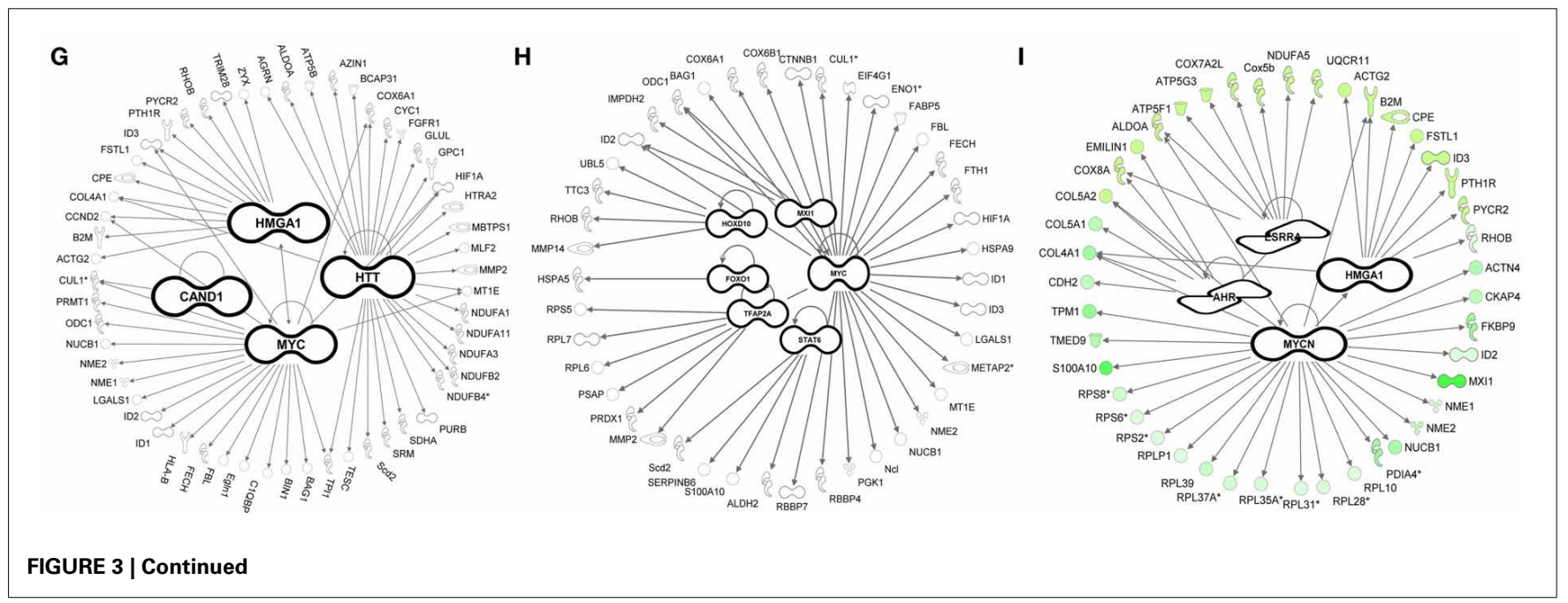


$\mathbf{J}$

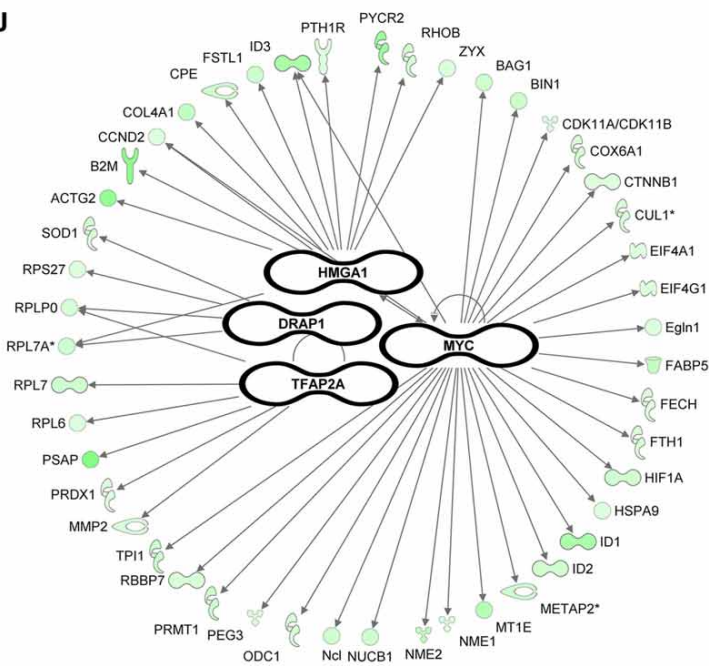

$\mathbf{K}$

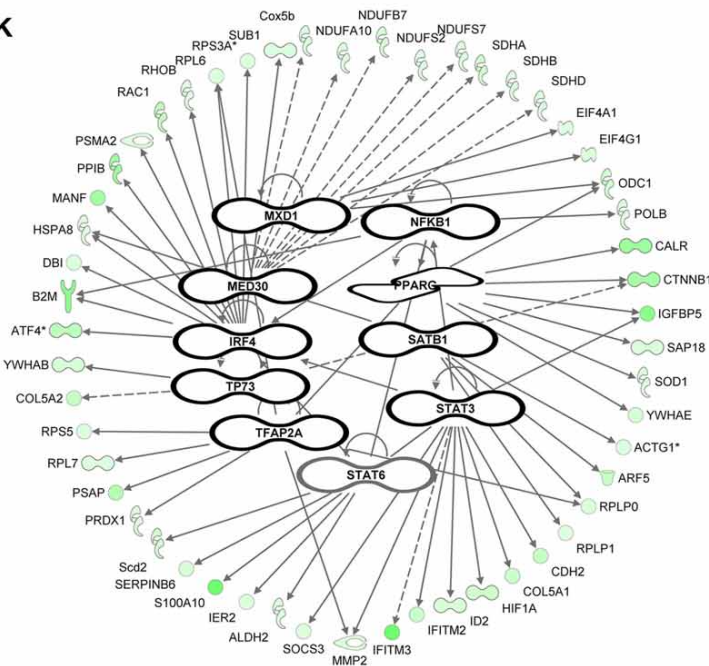

L

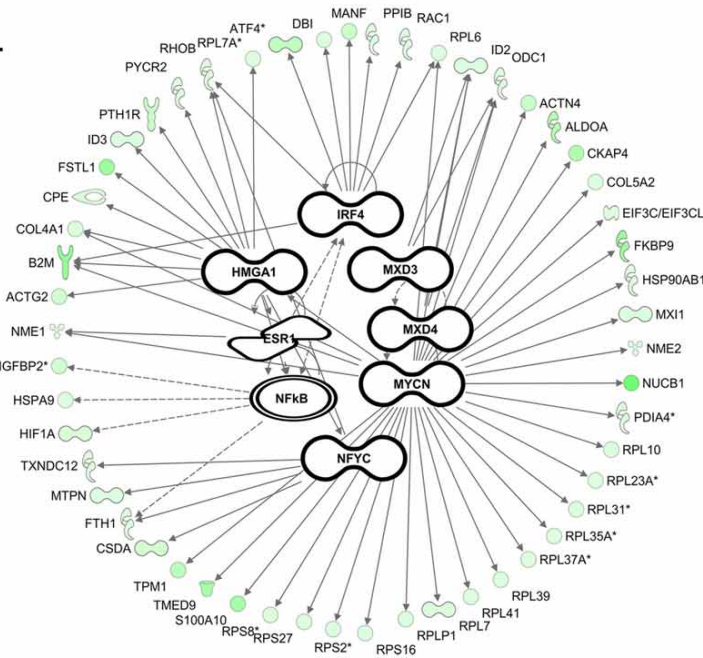

FIGURE 3 | Transcription factor analysis (IPA). Bioinformatics transcription factor analysis performed using Ingenuity Pathway Analysis (IPA) for the 4362 genes throughout the time-course (A-O) exhibiting a $p$-value of overlap $<0.01$ associating significantly $\sim 1000$ genes with 63 transcription factors. Pre-natal
M

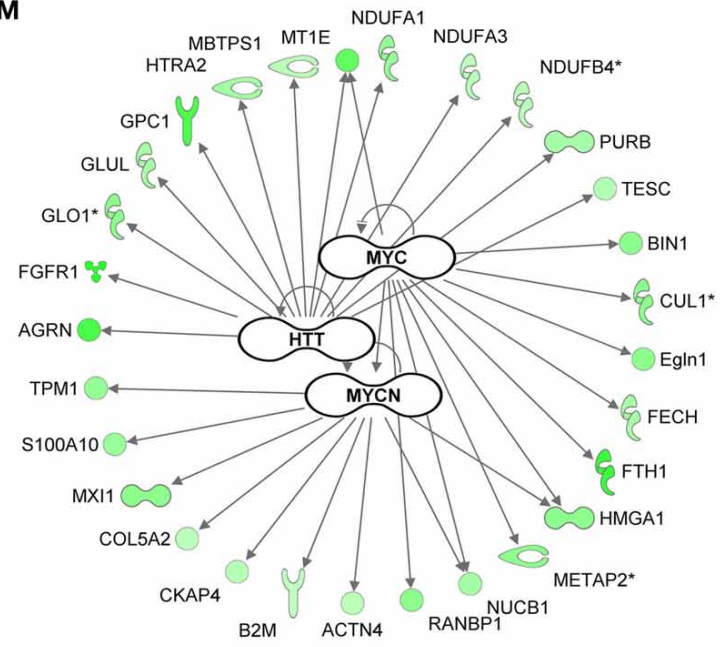

$\mathbf{N}$
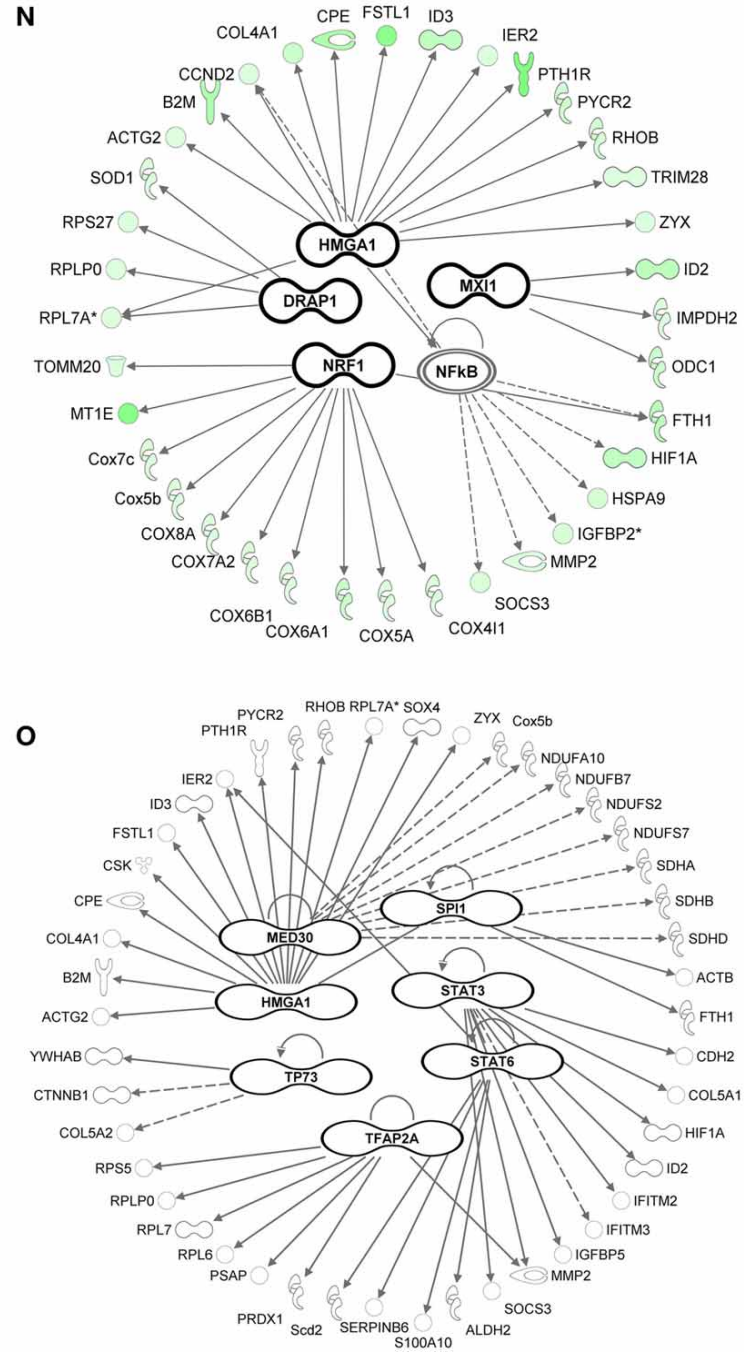

stages: Bud stages [A (E12.5), B (E13.5)]. Enamel knot stage [C (E14.5)]. Bell stages [D (E15.5), E (E16.5), F (E17.5), and G (E18.5)]. Day of birth (P0). Post-natal stages: Differentiation of odontoblasts (I-K), start of mineralization: dentin deposition (N-L), enamel deposition (0). 
Table 3 | Canonical pathways.

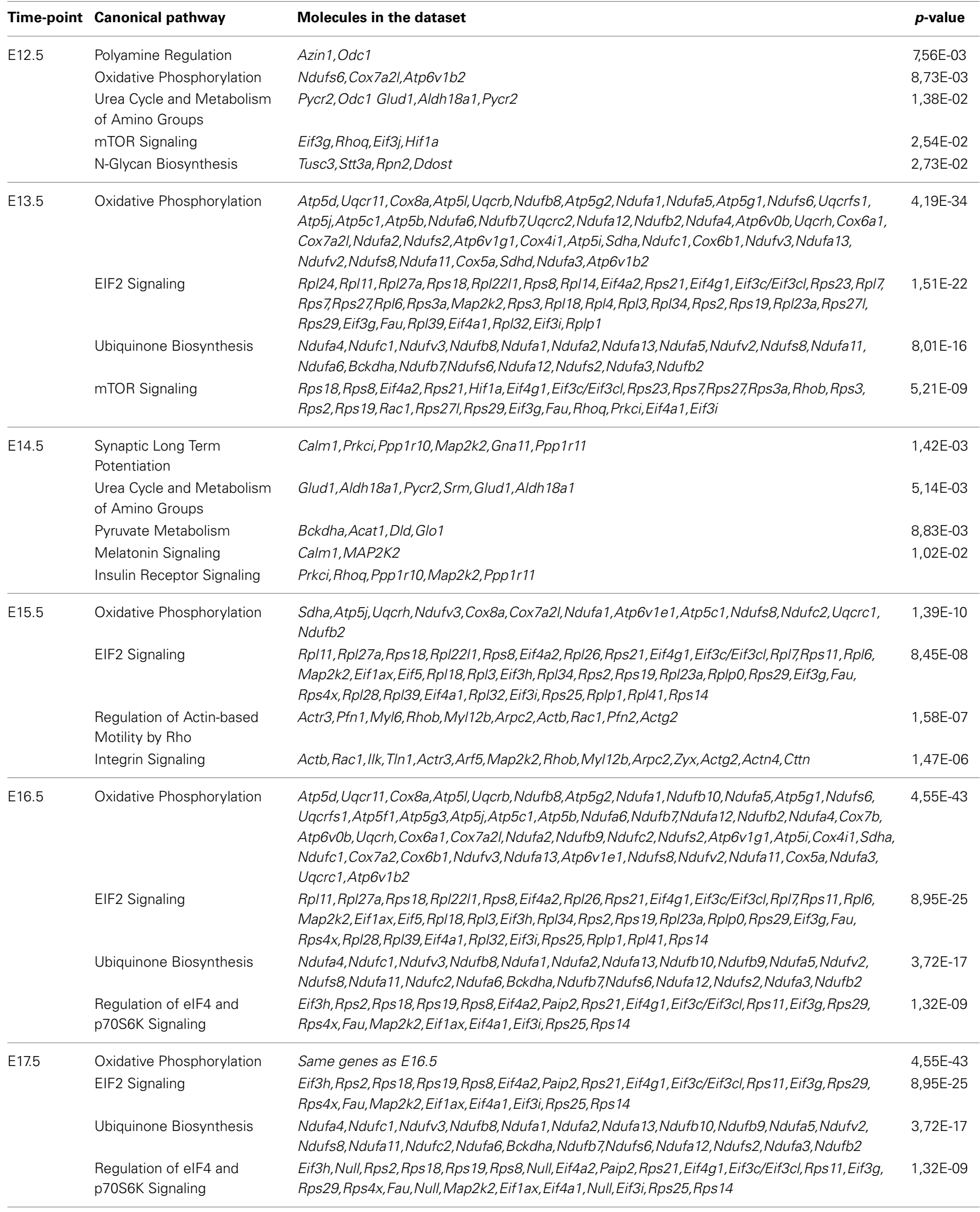


Table 3 | Continued

\begin{tabular}{|c|c|c|c|}
\hline Time-point & Canonical pathway & Molecules in the dataset & $p$-value \\
\hline \multirow{2}{*}{ E18.5 } & EIF2 Signaling & 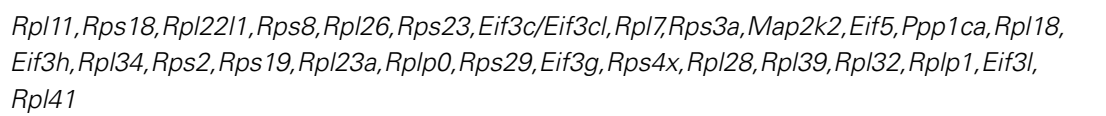 & 3,95E-19 \\
\hline & $\begin{array}{l}\text { Regulation of Actin-based } \\
\text { Motility by Rho }\end{array}$ & Pfn1,Cfl1, Myl6,Actb,Arpc5, Rac1,Pfn2,Actr3, Rhoq, Rhob,Arpc2,Arpc3,Actg2 & $1,66 \mathrm{E}-08$ \\
\hline \multirow[t]{4}{*}{ PO } & Oxidative Phosphorylation & 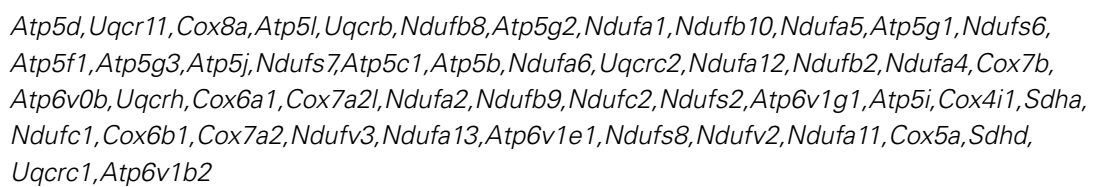 & $1,52 \mathrm{E}-44$ \\
\hline & Ubiquinone Biosynthesis & $\begin{array}{l}\text { Ndufa4,Ndufc1,Ndufs 7,Ndufv3, Ndufb8, Ndufa1, Ndufa2, Ndufa13, Ndufb 10, Ndufa5, Ndufb9, } \\
\text { Ndufv2,Ndufs8, Ndufa11,Ndufc2,Ndufa6, Bckdha, Ndufs6, Ndufa12,Ndufs2, Ndufb2 }\end{array}$ & $3,1 \mathrm{E}-17$ \\
\hline & $\begin{array}{l}\text { Regulation of Actin-based } \\
\text { Motility by Rho }\end{array}$ & Rhoq,Actr3, Pfn1, Rhob, Myl6, Cfl1, Myl12b,Arpc2,Actb, Pfn2, Rac1,Arpc3,Actg2 & $4,26 \mathrm{E}-26$ \\
\hline & $\begin{array}{l}\text { Regulation of elF4 and } \\
\text { p70S6K Signaling }\end{array}$ & $\begin{array}{l}\text { Rps18, Rps8, Eif4a2, Rps21, Eif4g1, Eif3c/Eif3cl,Rps23, Rps7,Rps27,Rps3a, Eif4g2,Map2k2, Eif1ax, } \\
\text { Rps3,Eif3h,Rps2,Rps19,Eif3j,Eif3g, Rps29, Rps4x,Fau,Eif3i,Rps25, Rps14 }\end{array}$ & $1,75 \mathrm{E}-13$ \\
\hline
\end{tabular}

P1 Oxidative Phosphorylatio

Atp6v0b, Uqcrh, Cox6a1, Uqcr11, Cox8a, Atp5I, Uqcrb, Cox7a21,Atp5g2, Ndufa1, Ndufb10, Ndufa5,

8,39E-31 Ndufb9,Atp6v1g1,Atp5f1,Atp5i,Cox4i1,Atp5g3,Sdha, Ndufc1,Cox7a2, Ndufv3,Ndufa13, Atp6v1e1,Ndufv2,Ndufs8, Ndufa11,Ndufa6, Uqcrc2, Cox5a,Ndufa3,Ndufb2,Atp6v1b2

Ubiquinone Biosynthesis

Ndufc1,Ndufv3, Ndufa1,Ndufa13,Ndufb10,Ndufa5,Ndufb9, Ndufs8, Ndufv2, Ndufa11,Ndufa6,

1,39E-10 Ndufa3,Ndufb2

EIF2 Signaling Rpl34, Rps2, Eif3j, Rps8, Rps21, Rps29, Map2k2, Rpl28, Rp/39, Eif5, Rpl32, Eif3i, Rplp1

$4,58 \mathrm{E}-07$

Integrin Signaling

Rapgef1,Capn6, Ralb, Ilk,Arf1,Actr3,Arf5, Rhoq, Rhob, Map2k2,Myl12b,Arpc2,Arpc3,Actg2,

2,56E-05 Actn4,Cttn

P2 Oxidative Phosphorylation

Atp5d, Uqcr11, Cox8a, Atp5I, Uqcrb, Ndufb8, Atp5g2, Ndufa1, Ndufb10, Ndufa5, Ndufs6, Uqcrfs1,

$5,12 \mathrm{E}-40$ Atp5f1,Atp5g3,Atp5j,Atp5c1,Atp5b, Ndufa6, Uqcrc2, Ndufa12,Ndufb2,Atp6v0b, Uqcrh, Cox6a1, Cox7a21, Ndufa2, Ndufb9, Ndufc2, Ndufs2, Atp6v1g1, Cox4i1, Atp5i,Sdha, Ndufc1, Cox7a2, Ndufv3, Ndufa13,Atp6v1e1,Ndufv2,Ndufs8, Ndufa11, Cox5a,Uqcrc1,Atp6v1b2

EIF2 Signaling Rp/11,Rp/27a, Eif4a2, Rps23,Eif3c/Eif3cl, Rps11,Rps7,Map2k2, Eif1ax, Ppp1ca, Pabpc1, Rpl3, Rpl23a, Rplp0, Rps29, Rps4x, Fau, Rpl41, Rplp1, Rpl24, Rps18, Rpl2211rps8, Rpl26, Rps21, Eif4g1, Rpl7,Rpl6, Rps27,Rps3, Rpl18, Rpl4, Rp/34, Rps2, Rps19, Eif3g, Rpl28, Eif4a1, Rpl32, Eif3I, Rps 14

Ubiquinone Biosynthesis Ndufc1, Null, Ndufv3,Null, Ndufb8, Ndufa1,Ndufa2, Ndufa13,Ndufb 10, Null, Ndufb9, Ndufa5, $8,16 \mathrm{E}-17$ Ndufv2, Ndufs8, Ndufa11, Ndufc2,Ndufa6, Bckdha,Ndufs6, Ndufa12,Ndufs2,Null,Ndufb2

Regulation of elF4 and Rhoq,Actr3, Pfn1,Rhob, Myl6, Cfl1,Myl12b,Arpc2,Actb, Null,Pfn2, Rac1,Arpc3,Actg2 p70S6K Signaling 
Table 3 | Continued

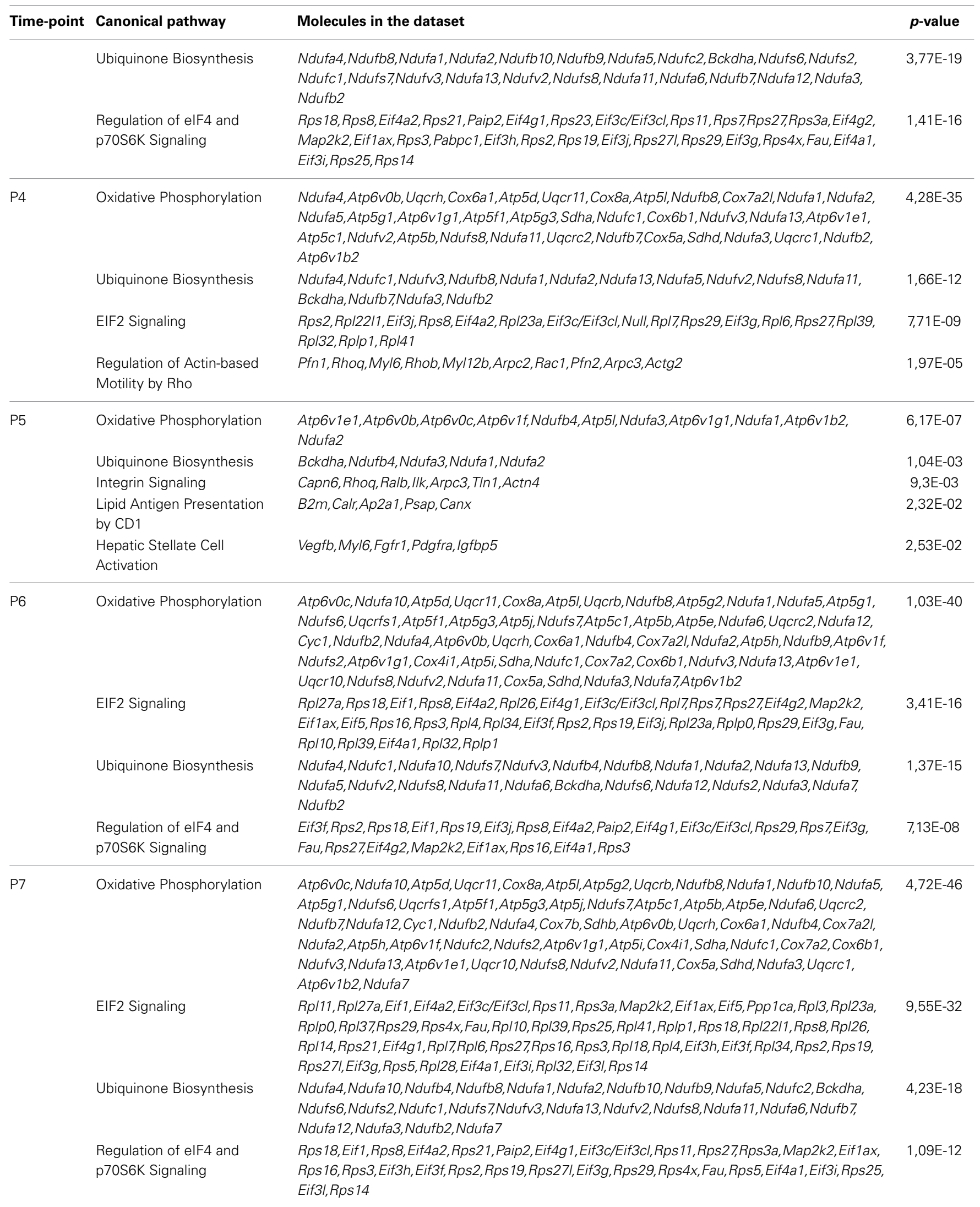

Signaling or metabolic pathways (IPA) significantly associated with DE genes during murine tooth development. 
Table 4 | Biological functions associated with network analysis.

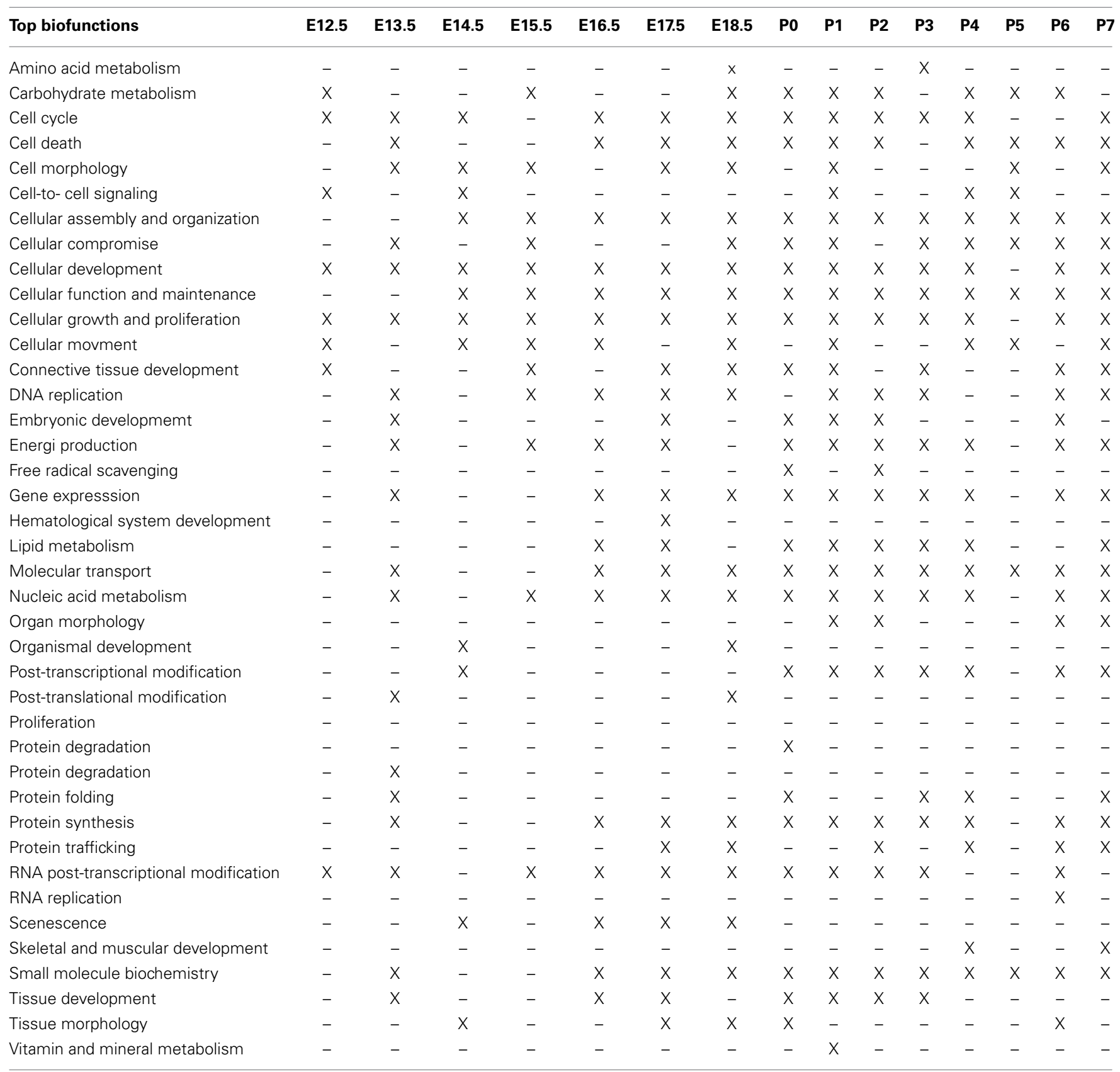

The table shows a summary of the biological functions significantly associated with the networks resulting from bioinformatics time-course network analysis presented as supplementary data.

regeneration allows the growing epithelial derived bud/bell to grow into and displace the mesenchymal tissue. This is probably modulated by e.g., Cdkn2a, Foxo, Smarcc, and TP53 (E12.5E15.5). $M y c N$ is the cytoplasmic form of Myc where interacts with $\alpha$ - and $\beta$-tubulins and is expressed in differentiating cells (Conacci-Sorrell et al., 2010). Transcription factor analysis suggests that $M y c N$ regulates different genes at the enamel knot stage E14.5 early bell stages E15.5-E16.5 and late bell stage (P0). At post natal stages P4-P5 $M y c N$ may in addition to participate in cell proliferation and control of cell cycle progression be involved in the differentiation of mesenchyme cells into odontoblasts.

Microarray and $\mathrm{qPCR}$ results suggest that metallotionin 1 $(\mathrm{Mt1})$ is highly expressed during the bell stage (E16.5) and postnatal stages (P5-P7). Very little is known about the expression of metallothionein 1 in the developing murine teeth prior to mineralization. Metallothioneins together with zinc transporters control zinc (Zn) homeostasis (Nartey et al., 1987). Zn is an essential trace element indispensable in cellular processes for embryonic and postnatal development in mammals (Kitabayashi et al., 

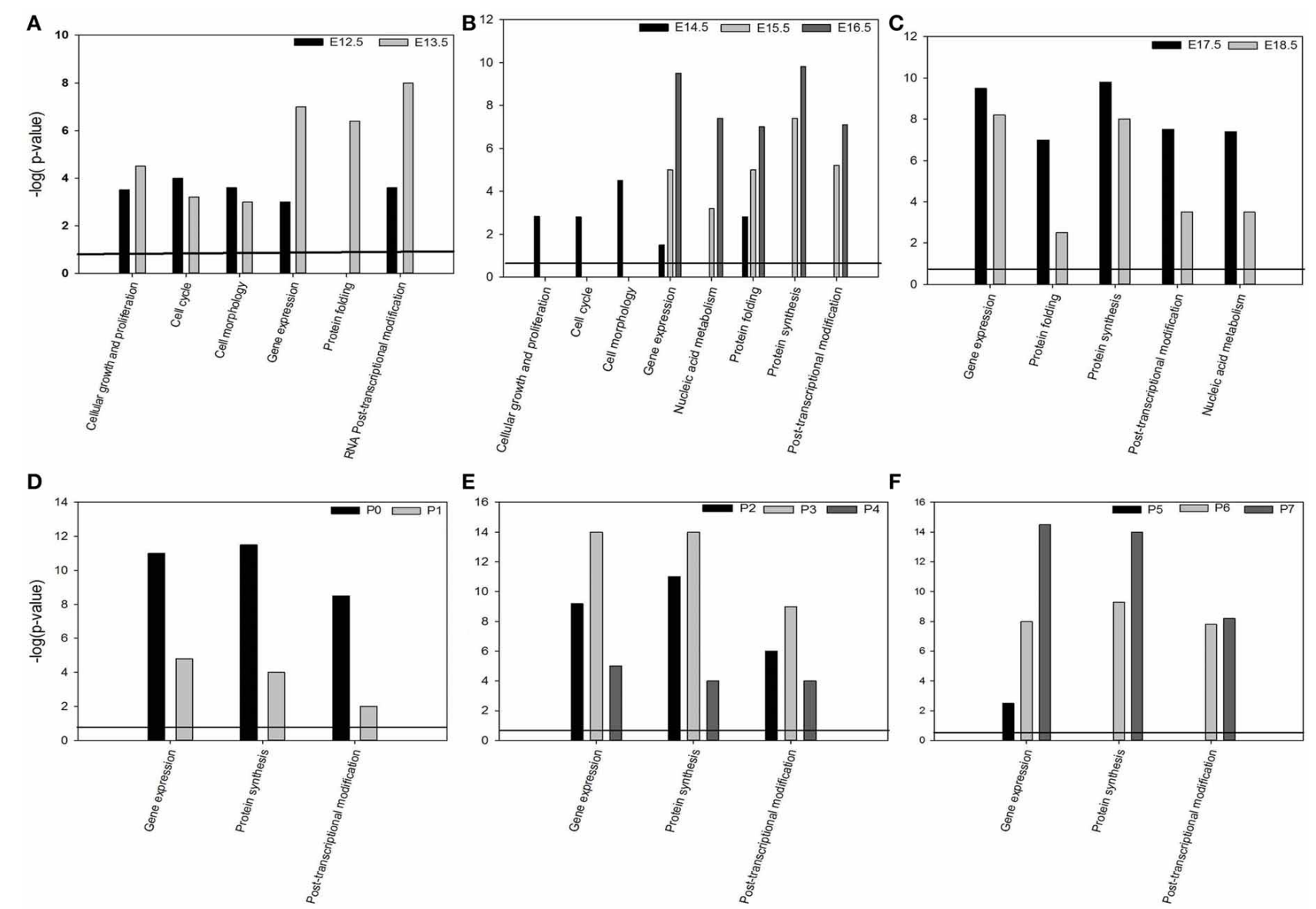

FIGURE 4 | Bioinformatics analysis of the global mRNA transcriptome during murine tooth development using Molecular \& Cellular functions (A-F) significantly $(p<0.005)$ associated with DE genes expressed during murine tooth development.

2010). Zn deficiency causes growth retardation, reduced bone volume, dental decay. Zinc equilibrium is also required for odontoblast differentiation and dentin formation during dentinogenesis (Lin et al., 2011).

During differentiation stages (Wang et al., 2013) (P0-P5) and early mineralization stages (P6-P7) in addition to cell migration apoptosis and cell death, mesenchymal cells facing the epithelial cells at the basal side of the growing bell start to differentiate, elongate following by secretion of dentin around P4-P5, triggering secretion and mineralization initiation by the epithelial cells now differentiating into ameloblasts (P6-P7). Some transcription factors like Myc, Mycn, Htt Stat6, Mxi1, and Drap1 seem to regulate different genes at the post-natal stages (P0, P2, P4, P5, and P6) compared to the embryonic stages (E12.5, E13.5, and E16.5). Esr1 (P4) encode for an estrogen receptor. Estrogen and its receptors are essential for epithelial cell development, proliferation, suggesting that not only genes and their products but also hormones play an important during murine tooth development (Wang et al., 2013). Low estrogen production is associated with increased production of $T g f a$, interleukins $1,6,8$, and 10 leading to periodontal disease (Tezal et al., 2000; Dvorak et al., 2009; Zhang et al., 2011).
It is important to point out that levels of mRNA do not necessarily correlate with levels of translated protein (protein biosynthesis) from the same mRNA (Pascal et al., 2008). The quantitation of levels of mRNA and protein are complementary and also necessary for a complete understanding of how altered gene expression affects cellular physiology (Greenbaum et al., 2003), in addition to miRNAs, long non-coding RNAs (lncRNAs) (Okazaki et al., 2002; Batista and Chang, 2013) that do not code for functional proteins (Rinn and Chang, 2012) may play an important role in spatial positioning of the epithelial/mesenchymal cells during murine tooth development adding layers of complexity to the study of murine tooth development.

\section{AUTHOR CONTRIBUTIONS}

ML performed microarray, RT-PCR and bioinformatics analysis. Prof. HO provided exceptional scientific resources, guidance and incredible scientific inspiration. MS helped with qPCR. SN contributed with bioinformatics expertise. EB performed qPCR oligoprimers synthesis and analysis. JR helped with fruitfully discussions editing the manuscript. 


\section{ACKNOWLEDGMENTS}

The technical skills of Ms. Toril Woldene were highly appreciated. I also thank the staff of the animal House facility; Mr. Benedicto Geronimo and Mr. Nikanor Aggabao for invaluable technical support during dissection of tooth germs and for maintain our stock of mice.

\section{SUPPLEMENTARY MATERIAL}

The Supplementary Material for this article can be found online at: http://www.frontiersin.org/journal/10.3389/fgene. 2015.00047/abstract

\section{REFERENCES}

Ashburner, M., Ball, C. A., Blake, J. A., Botstein, D., Butler, H., Cherry, J. M., et al. (2000). Gene ontology: tool for the unification of biology. The Gene Ontology. Consortium. Nat Genet. 25, 25-29. doi: 10.1038/75556

Batista, P. J., and Chang, H. Y. (2013). Long noncoding RNAs: cellular address codes in development and disease. Cell 152, 1298-1307. doi: 10.1016/j.cell.2013.02.012

Benjamini, Y., Drai, D., Elmer, G., Kafkafi, N., and Golani, I. (2001). Controlling the false discovery rate in behavior genetics research. Behav. Brain Res. 125, 279-284. doi: 10.1016/S0166-4328(01)00297-2

Cheadle, C., Vawter, M. P., Freed, W. J., and Becker, K. G. (2003). Analysis of microarray data using $\mathrm{Z}$ score transformation. J. Mol. Diagn. 5, 73-81. doi: 10.1016/S1525-1578(10)60455-2

Conacci-Sorrell, M., Ngouenet, C., and Eisenman, R. N. (2010). Myc-nick: a cytoplasmic cleavage product of Myc that promotes alpha-tubulin acetylation and cell differentiation. Cell 142, 480-493. doi: 10.1016/j.cell.2010. 06.037

Dvorak, G., Reich, K., Tangl, S., Lill, C. A., Gottschalk-Baron, M., Watzek, G., et al. (2009). Periodontal histomorphometry and status of aged sheep subjected to ovariectomy, malnutrition and glucocorticoid application. Arch. Oral Biol. 54, 857-863. doi: 10.1016/j.archoralbio.2009.05.010

Etokebe, G. E., Kuchler, A. M., Haraldsen, G., Landin, M., Osmundsen, H., and Dembic, Z. (2009). Family-with-sequence-similarity-46, member A (Fam46a) gene is expressed in developing tooth buds. Arch. Oral Biol. 54, 1002-1007. doi: 10.1016/j.archoralbio.2009.08.005

Greenbaum, D., Colangelo, C., Williams, K., and Gerstein, M. (2003). Comparing protein abundance and mRNA expression levels on a genomic scale. Genome Biol. 4:117. doi: 10.1186/gb-2003-4-9-117

Haara, O., Harjunmaa, E., Lindfors, P. H., Huh, S. H., Fliniaux, I., Aberg, T., et al. (2012). Ectodysplasin regulates activator-inhibitor balance in murine tooth development through Fgf20 signaling. Development 139, 3189-3199. doi: 10.1242/dev.079558

Ikuta, J., Kamisetty, N. K., Shintaku, H., Kotera, H., Kon, T., and Yokokawa, R. (2014). Tug-of-war of microtubule filaments at the boundary of a kinesin- and dynein-patterned surface. Sci. Rep. 4:5281. doi: 10.1038/srep05281

Jevnaker, A. M., and Osmundsen, H. (2008). MicroRNA expression profiling of the developing murine molar tooth germ and the developing murine submandibular salivary gland. Arch. Oral Biol. 53, 629-645. doi: 10.1016/j.archoralbio.2008.01.014

Kitabayashi, C., Fukada, T., Kanamoto, M., Ohashi, W., Hojyo, S., Atsumi, T., et al. (2010). Zinc suppresses Th17 development via inhibition of STAT3 activation. Int. Immunol. 22, 375-386. doi: 10.1093/intimm/dxq017

Kramer, A., Green, J., Pollard, J. Jr., and Tugendreich, S. (2014). Causal analysis approaches in Ingenuity Pathway Analysis. Bioinformatics 30, 523-530. doi: 10.1093/bioinformatics/btt703

Landin, M. A., Shabestari, M., Babaie, E., Reseland, J. E., and Osmundsen, H. (2012). Gene expression profiling during murine tooth development. Front. Genet. 3:139. doi: 10.3389/fgene.2012.00139

Lin, C. Y., Lin, H. H., Tsai, M. H., Lin, S. P., and Chen, M. H. (2011). Zinc chloride for odontogenesis of dental pulp stem cells via metallothionein up-regulation. J. Endod. 37, 211-216. doi: 10.1016/j.joen.2010.11.009

Liu, F., and Millar, S. E. (2010). Wnt/beta-catenin signaling in oral tissue development and disease. J. Dent. Res. 89, 318-330. doi: 10.1177/00220345103 63373
Liu, L., Ling, J., Wei, X., Wu, L., and Xiao, Y. (2009). Stem cell regulatory gene expression in human adult dental pulp and periodontal ligament cells undergoing odontogenic/osteogenic differentiation. J. Endod. 35, 1368-1376. doi: 10.1016/j.joen.2009.07.005

Michon, F. (2011). Tooth evolution and dental defects: from genetic regulation network to micro-RNA fine-tuning. Birth Defects Res. A Clin. Mol. Teratol. 91, 763-769. doi: 10.1002/bdra.20787

Michon, F., Tummers, M., Kyyronen, M., Frilander, M. J., and Thesleff, I. (2010). Tooth morphogenesis and ameloblast differentiation are regulated by micro-RNAs. Dev. Biol. 340, 355-368. doi: 10.1016/j.ydbio.2010. 01.019

Morsczeck, C., Schmalz, G., Reichert, T. E., Vollner, F., Saugspier, M., VialeBouroncle, S., et al. (2009). Gene expression profiles of dental follicle cells before and after osteogenic differentiation in vitro. Clin. Oral Investig. 13, 383-391. doi: 10.1007/s00784-009-0260-x

Munoz-Espin, D., and Serrano, M. (2014). Cellular senescence: from physiology to pathology. Nat. Rev. Mol. Cell. Biol. 15, 482-496. doi: 10.1038/nrm 3823

Nartey, N. O., Banerjee, D., and Cherian, M. G. (1987). Immunohistochemical localization of metallothionein in cell nucleus and cytoplasm of fetal human liver and kidney and its changes during development. Pathology 19, 233-238. doi: 10.3109/00313028709066555

Okazaki, Y., Furuno, M., Kasukawa, T., Adachi, J., Bono, H., Kondo, S., et al. (2002). Analysis of the mouse transcriptome based on functional annotation of 60,770 full-length cDNAs. Nature 420, 563-573. doi: 10.1038/nature 01266

Osmundsen, H., Landin, M. A., From, S. H., Kolltveit, K. M., and Risnes, S. (2007). Changes in gene-expression during development of the murine molar tooth germ. Arch. Oral Biol. 52, 803-813. doi: 10.1016/j.archoralbio.2007. 02.008

Pascal, L. E., True, L. D., Campbell, D. S., Deutsch, E. W., Risk, M., Coleman, I. M. et al. (2008). Correlation of mRNA and protein levels: cell type-specific gene expression of cluster designation antigens in the prostate. BMC Genomics 9:246. doi: 10.1186/1471-2164-9-246

Pfaffl, M. W., Georgieva, T. M., Georgiev, I. P., Ontsouka, E., Hageleit, M., and Blum, J. W. (2002a). Real-time RT-PCR quantification of insulin-like growth factor (IGF)-1, IGF-1 receptor, IGF-2, IGF-2 receptor, insulin receptor, growth hormone receptor, IGF-binding proteins 1, 2 and 3 in the bovine species. Domest. Anim. Endocrinol. 22, 91-102. doi: 10.1016/S0739-7240(01) 00128-X

Pfaffl, M. W., Horgan, G. W., and Dempfle, L. (2002b). Relative expression software tool (REST) for group-wise comparison and statistical analysis of relative expression results in real-time PCR. Nucleic Acids Res. 30:e36. doi: 10.1093/nar/30.9.e36

Quackenbush, J. (2002). Microarray data normalization and transformation. Nat. Genet. 32(Suppl.), 496-501. doi: 10.1038/ng1032

Reiner, A., Yekutieli, D., and Benjamini, Y. (2003). Identifying differentially expressed genes using false discovery rate controlling procedures. Bioinformatics 19, 368-375. doi: 10.1093/bioinformatics/btf877

Rhee, S. Y., Wood, V., Dolinski, K., and Draghici, S. (2008). Use and misuse of the gene ontology annotations. Nat. Rev. Genet. 9, 509-515. doi: 10.1038/nrg 2363

Rinn, J. L., and Chang, H. Y. (2012). Genome regulation by long noncoding RNAs. Annu. Rev. Biochem. 81, 145-166. doi: 10.1146/annurev-biochem-051410092902

Smyth, G. K. (2004). Linear models and empirical bayes methods for assessing differential expression in microarray experiments. Stat. Appl. Genet. Mol. Biol. 3:3. doi: 10.2202/1544-6115.1027

Tezal, M., Wactawski-Wende, J., Grossi, S. G., Ho, A. W., Dunford, R., and Genco, R. J. (2000). The relationship between bone mineral density and periodontitis in postmenopausal women. J. Periodontol. 71, 1492-1498. doi: 10.1902/jop.2000.71.9.1492

Thomas, S., and Bonchev, D. (2010). A survey of current software for network analysis in molecular biology. Hum. Genomics 4, 353-360. doi: 10.1186/14797364-4-5-353

Wang, Y., Yan, M., Yu, Y., Wu, J., Yu, J., and Fan, Z. (2013). Estrogen deficiency inhibits the odonto/osteogenic differentiation of dental pulp stem cells via activation of the NF-kappaB pathway. Cell Tissue Res. 352, 551-559. doi: 10.1007/s00441-013-1604-z 
Zhang, B., Li, Y., Zhou, Q., and Ding, Y. (2011). Estrogen deficiency leads to impaired osteogenic differentiation of periodontal ligament stem cells in rats. Tohoku J. Exp. Med. 223, 177-186. doi: 10.1620/tjem. 223.177

Conflict of Interest Statement: The authors declare that the research was conducted in the absence of any commercial or financial relationships that could be construed as a potential conflict of interest.

Received: 16 December 2014; accepted: 02 February 2015; published online: 26 February 2015.
Citation: Landin MA, Nygård S, Shabestari MG, Babaie E, Reseland JE and Osmundsen $H$ (2015) Mapping the global mRNA transcriptome during development of the murine first molar. Front. Genet. 6:47. doi: 10.3389/fgene.2015.00047

This article was submitted to Systems Biology, a section of the journal Frontiers in Genetics.

Copyright (c) 2015 Landin, Nygård, Shabestari, Babaie, Reseland and Osmundsen. This is an open-access article distributed under the terms of the Creative Commons Attribution License (CC BY). The use, distribution or reproduction in other forums is permitted, provided the original author(s) or licensor are credited and that the original publication in this journal is cited, in accordance with accepted academic practice. No use, distribution or reproduction is permitted which does not comply with these terms. 